\title{
El taladrador de la uva de mar, Hexeris enhydris Grote (Insecta: Lepidoptera: Thyrididae) ${ }^{1}$
}

Forrest W. Howard ${ }^{2}$

\section{Introducción}

El taladrador de la uva de mar, Hexeris enhydris, es una especie de polilla cuya larva taladra las ramas delgadas de la uva de mar, Coccoloba uvifera L., y una especie cercanamente relacionada, uvilla, $C$. diversifolia Jacquin (Polygonaceae). La uva de mar es un árbol pequeño o arbusto y la uvilla es un árbol algo grande al alcanzar madurez. Ambas especies crecen naturalmente en ambientes costeras en la Florida y la región del Caribe. La uva de mar, y en un grado inferior la uvilla, son frecuentemente cultivadas en los jardines ornamentales urbanos en el sur de la Florida y en muchos países Caribeños.

\section{Distribución}

El taladrador de uva de mar se encuentra a través del rango de la uva de mar en la Florida, por ejemplo, en las áreas costeras del centro y sur de la Florida y en los Cayos (Chellman 1978). El taladrador también ha sido encontrado en Cuba (Grote 1875). Este es quizás mas ampliamente distribuido a través del Caribe de lo que ha sido reportado, ya que la misma uva de mar está presente en zonas costeras de la región, y el género

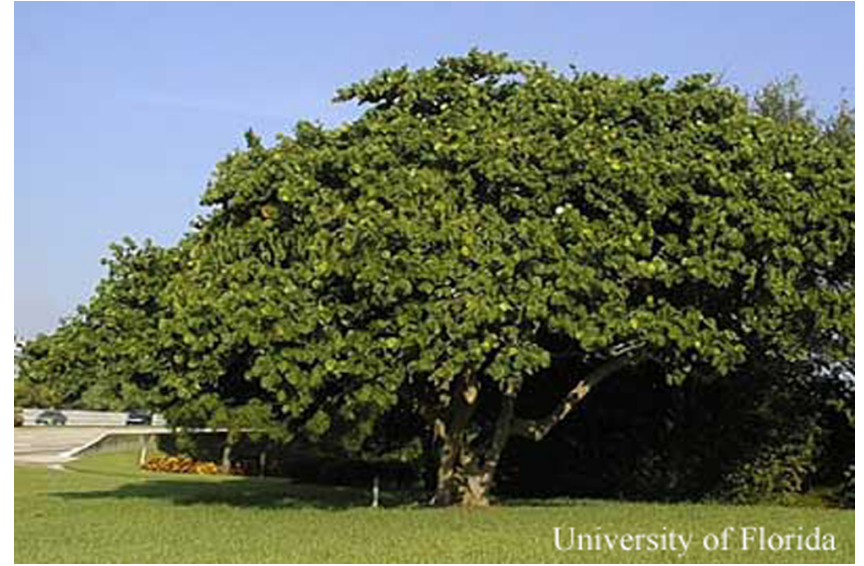

Figure 1. La uva de mar, Coccoloba uvifera L. Credits: F.W. Howard, University of Florida

Coccoloba contiene unas 180 especies distribuidas en América Tropical, y algunas de estas posiblemente pueden ser huéspedes no reportados.

\section{Apariencia}

Adulto: La polilla adulta es de color amarillento a café pálido con líneas onduladas de color de ladrillo en las alas delanteras y con una envergadura de 34 a $38 \mathrm{~mm}$. Los prominentes palpos labiales se extienden hacia adelante una distancia igual a dos veces del largo de la cabeza.

1. This document is EENY-347, one of a series of Featured Creatures from the Entomology and Nematology Department, Florida Cooperative Extension Service, Institute of Food and Agricultural Sciences, University of Florida. Published: April 2005. This document is also available on Featured Creatures Website at http://creatures.ifas.ufl.edu. Please visit the EDIS Website at http://edis.ifas.ufl.edu.

2. Forrest W. Howard, Department of Entomology and Nematology, University of Florida. Traductores: F. W. Howard and Juan Sebastián Ortiz.

The Institute of Food and Agricultural Sciences (IFAS) is an Equal Opportunity Institution authorized to provide research, educational information and other services only to individuals and institutions that function with non-discrimination with respect to race, creed, color, religion, age, disability, sex, sexual orientation, marital status, national origin, political opinions or affiliations. U.S. Department of Agriculture, Cooperative Extension Service, University of Florida, IFAS, Florida A. \& M. University Cooperative Extension Program, and Boards of County Commissioners Cooperating. Larry Arrington, Dean 


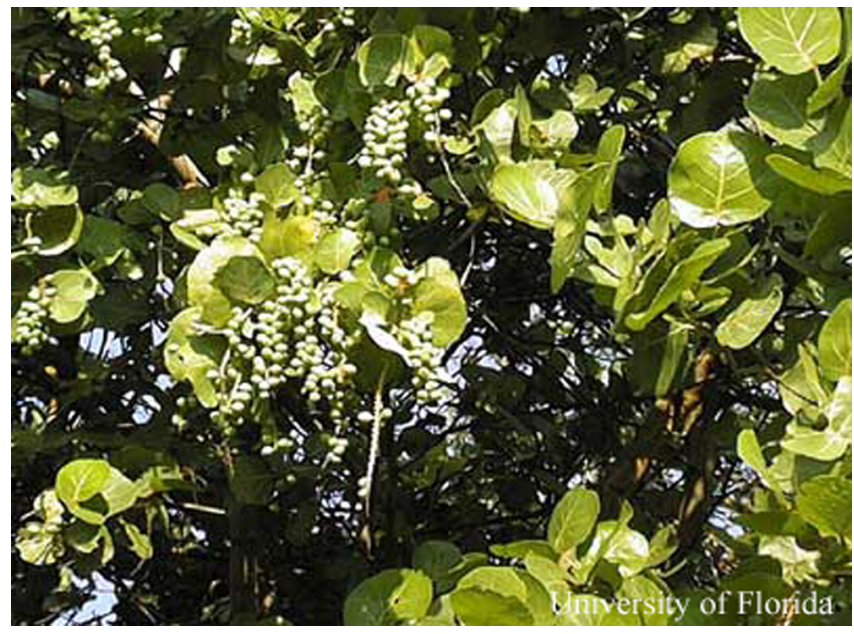

Figure 2. La uva de mar, Coccoloba uvifera L., vista de cerca. Credits: F.W. Howard, University of Florida

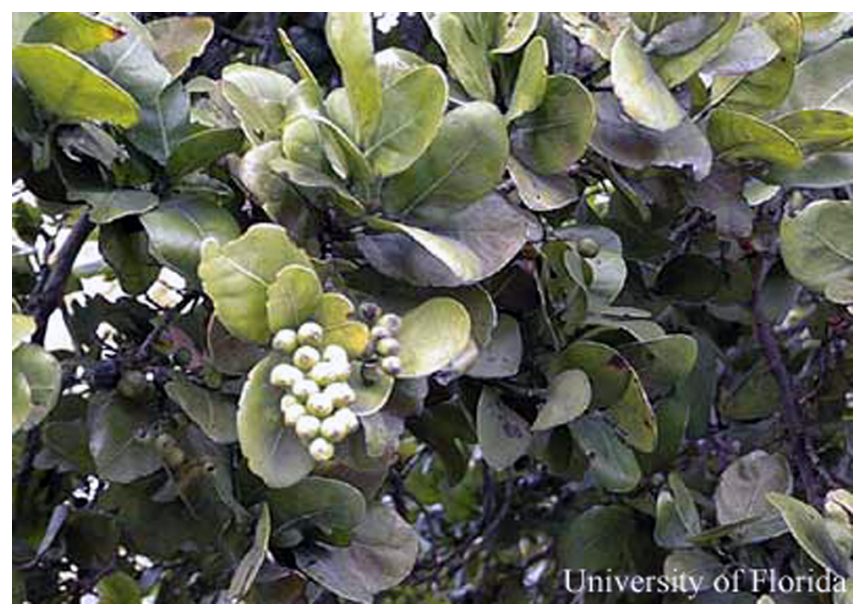

Figure 3. La uvilla, Coccoloba diversifolia Jacquin. Credits: F.W. Howard, University of Florida

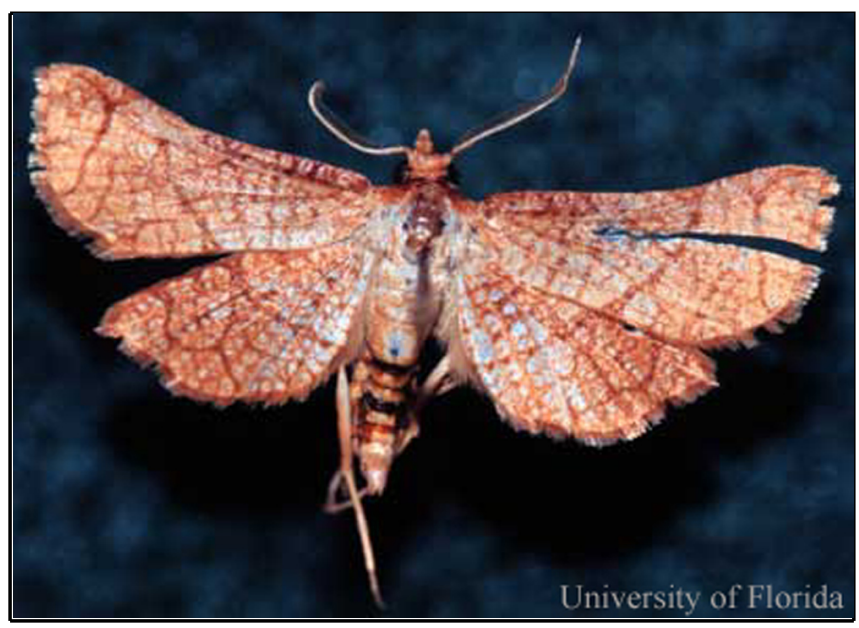

Figure 4. El taladrador de uva de mar, Hexeris enhydris Grote, adulto. Credits: F.W. Howard, University of Florida

Larva: Las larvas son apodas (sin patas), lo cual es una condición típica de las larvas taladradoras de madera. La larva madura es de aproximadamente $15 \mathrm{~mm}$ de largo.

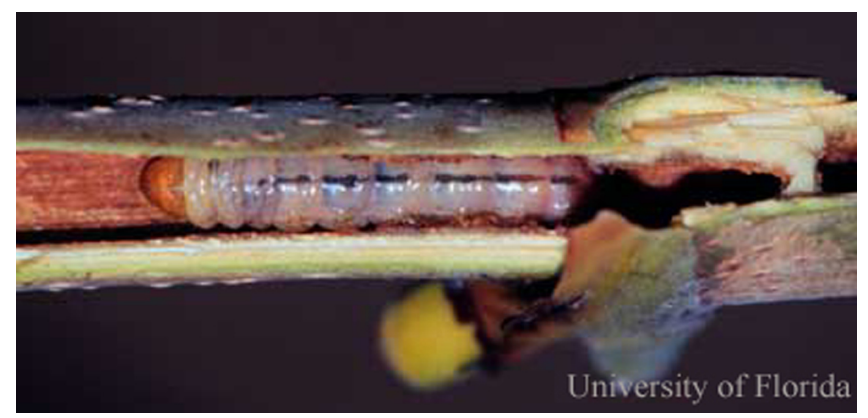

Figure 5. El taladrador de uva de mar, Hexeris enhydris Grote, rama partida para revelar larva. Credits: F.W. Howard, University of Florida

Pupa: Las pupas son café y casi igual de largas a las larvas maduras (Solomon 1995).

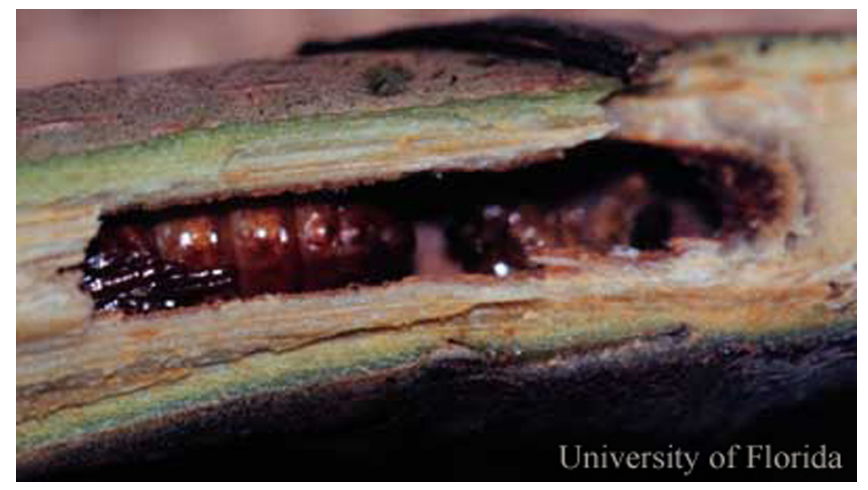

Figure 6. El taladrador de uva de mar, Hexeris enhydris Grote, rama partida para revelar pupa. Credits: F.W. Howard, University of Florida

\section{Biología}

Las polillas adultas han sido vistas volando durante los meses frescos igual como durante los meses calientes en la Florida (Chellman 1978). Presuntamente, las hembras ovipositan en las superficies de las ramas delgadas y las hojas, ya que los Lepidoptera no tienen ovipositores que puedan perforar los tejidos de la planta para insertar a los huevos. La larva probablemente se alimente en el exterior por un corto periodo al nacer antes de taladrar dentro del tejido de la planta. Taladra la rama delgada o pecíolo o raramente en la vena central de la hoja. Estas a veces cavan pequeños huecos en las paredes de los túneles hacia el exterior, los cuales son usados para expulsar los residuos de madera (Solomon 1995). La duración del desarrollo de la larva no es conocida. La transformación a pupa se 
lleva a cabo dentro del túnel. Los taladradores se han observado y colectado probablemente más a menudo de la uva de mar que de la uvilla, puesto que el último es menos común como planta ornamental. Cuando las polillas adultas emergen de la cápsula de la pupa, encuentran su ruta hacia una salida, salen del túnel, y vuelan para encontrar una pareja. Los insectos Lepidoptera se alimentan generalmente de néctar, pero aun no se ha determinado como se alimentan las polillas adultas de los taladradores de uva de mar.

\section{Daño}

Las ramas que han sido ahuecadas por los taladradores de uva de mar son fáciles de reconocer ya que ha a menudo el daño da como resultado la muerte de la hoja del tallo dañado. Las hojas de la uva de mar son grandes, típicamente de $16-20 \mathrm{~cm}$ de largo y ancho, y por esta razón las hojas muertas, las cuales vuelven amarillas a rojizas, (conocidas como "banderas") pueden ser vistas a larga distancia como indicadores del daño del taladrador. Las ramas que contienen taladradores, usualmente tienen áreas necróticas y huecos con residuos de madera saliendo de ellos. El partir la rama revele la pupa o la larva. Si el insecto ha completado su ciclo de desarrollo, una cápsula pupal vacía estará presente. El daño viejo casi siempre se constituye de ramas muertas vacías que están deshilachadas en el terminal (Johnson y Lyon 1991, Solomon 1995).

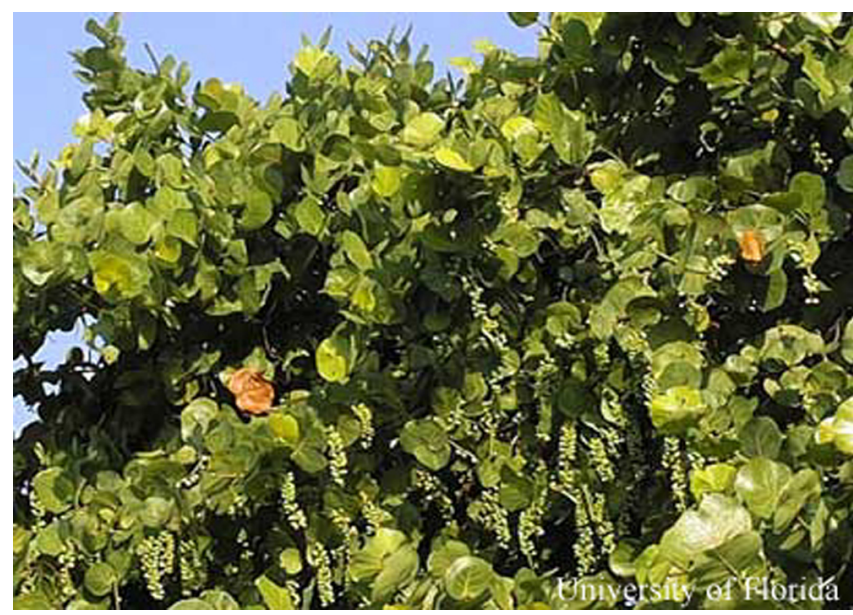

Figure 7. Uva de mar, Coccoloba uvifera L. Note dos hojas rojizas ("banderas"), los cuales son indicador del daño a ramas del taladrador de uva de mar, Hexeris enhydris Grote. Credits: F.W. Howard, University of Florida

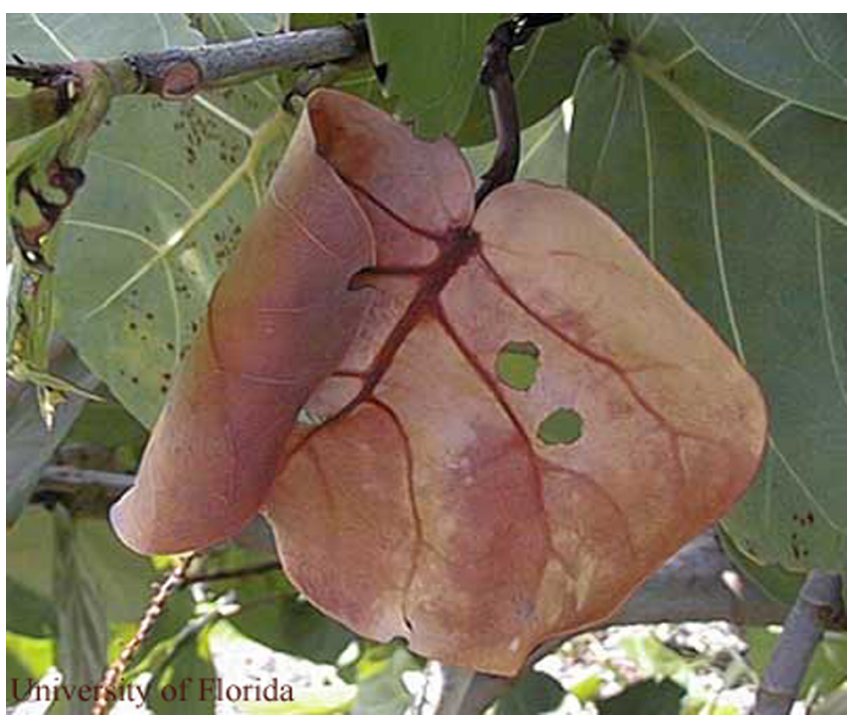

Figure 8. Uva de mar, Coccoloba uvifera L. Vista de cerca de hoja rojiza ("bandera") indicador del daño a la rama del taladrador de uva de mar, Hexeris enhydris Grote. Credits: F.W. Howard, University of Florida

\section{Perspectivas de Manejo}

Por lo menos una especies no identificada de Himenóptera parasítica ataca al taladrador de uva de mar (Moon y Stiling 2004), y es indudablemente debido a los enemigos naturales y otros factores que el taladrador usualmente es presente a niveles de población tolerables. Aunque está ampliamente distribuida sobre árboles de uva de mar, usualmente un porcentaje pequeño de tallos de estos árboles son atacados por el taladrador, y el daño, aunque sea conspicuo a los que lo que conocen, no es notado por la mayoría de la gente. Es solamente en casos raros que los taladradores se abundan y causan la muerte masiva de las ramas. El daño causado por este taladrador puede ser más serio en los árboles pequeños de viveros. El podar y destruir las ramas que contienen los taladradores ha sido recomendados como métodos para reducir las poblaciones (Chellman 1978), pero la efectividad de estos métodos no ha sido evaluada. Asimismo, el control con insecticidas no ha sido evaluado en años recientes.

\section{Agradecimientos}

Agradezco a los Drs. Robert Pemberton de USDA-ARS, Invasive Plants Laboratory, Fort Lauderdale, y William Kern, University of Florida, 
IFAS, Fort Lauderdale Research \& Education Center, por su revisión y comentos sobre el manuscrito.

\section{Referencias seleccionadas}

Chellman CW. 1978. Pests and problems of south Florida trees and palms. Tallahassee, Florida. Florida Department of Agriculture and Consumer Services, Division of Forestry.

Grote AR. 1875. On certain species of moths from Florida. Canadian Entomologist 7: 173-176.

Johnson WT, Lyon HH. 1991. Insects that feed on trees and shrubs. Ithaca and London, Comstock Publishing Associates, Cornell University Press.

Moon DC, Stiling P. 2004. The influence of a salinity and nutrient gradient on coastal vs. upland tritrophic complexes. Ecology 85: 2709-2716.

Solomon JD. 1995. Guide to insect borers of North American broadleaf trees and shrubs. Washington, D.C., U. S. Forest Service, U. S. Department of Agriculture. 